\title{
Pharmacovigilance of biosimilars: challenges and possible solutions
}

\author{
Thijs J Giezen, PharmD, PhD; Sabine MJM Straus, MD, PhD
}

Post-marketing surveillance is essential to detect, assess and prevent adverse reactions of chemically synthesized small molecule drugs as well as biologicals, as the full safety profile can only be known after they have been placed on the market. Biologicals have specific characteristics, which pose additional challenges in pharmacovigilance.

Keywords: Biologicals, biosimilars, immunogenicity, pharmacovigilance, traceability

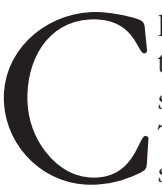

hallenges are encountered during the pharmacovigilance of biosimilars, including traceability. These challenges and possible solutions were presented at the Conference of the Drug Information Association (DIA) in Copenhagen, Denmark, in March 2012. This paper provides a summary of the presentation given at the DIA.

Post-marketing collected safety data offers a valuable and necessary complement to clinical trials [1]. This applies to both chemically synthesized small molecule drugs and biologicals. However, compared to chemically synthesized drugs, biologicals have specific characteristics which might complicate their safety assessment: 1) biologicals are often indicated for rare diseases, where it is difficult to include sufficient patients in the pre-approval clinical trials and the continuous assessment of the benefit-risk in the post-marketing setting will be more important [2]; 2) obtaining exposure data after approval can be challenging for biologicals, since they are quite often used only in the hospital setting. Population-based databases mainly include information from general practitioners (GPs) and public pharmacies and will, therefore, contain limited information on patients exposed to biologicals. In addition, biologicals are often used in multiple indications with different dosage regimens. This might further complicate the exposure assessment of biologicals and a different approach towards the estimation of the number of patients exposed is warranted [3]; 3) it is sometimes difficult to define the 'at-risk window', which is the period that a certain adverse event should be attributed to the drug, for biologicals, due to their often prolonged pharmacodynamic effects [4]; 4) biologicals are often indicated as secondor third-line therapy limiting their use to patients with more severe disease or worse prognosis after the failure of 'standard treatment'. In addition, this group of patients is often treated with concomitant medication and is often suffering from other diseases. For this reason channelling bias can easily occur [3]. From the above mentioned, it is clear that pharmacovigilance for biologicals poses additional challenges as compared to small molecules. These challenges apply to all biologicals, including biosimilars. However, for biosimilars additional challenges might be encountered. This paper aims to describe challenges in the pharmacovigilance of biosimilars and provide possible solutions to improve the pharmacovigilance of biosimilars.

\section{Pharmacovigilance of biosimilars}

At moment of regulatory approval of a biosimilar there is extensive information available on the reference biological. For the biosimilar specific data is limited to the comparability exercise. It is known that (small) changes in the production and purification process of biologicals can have (major) implications on their safety

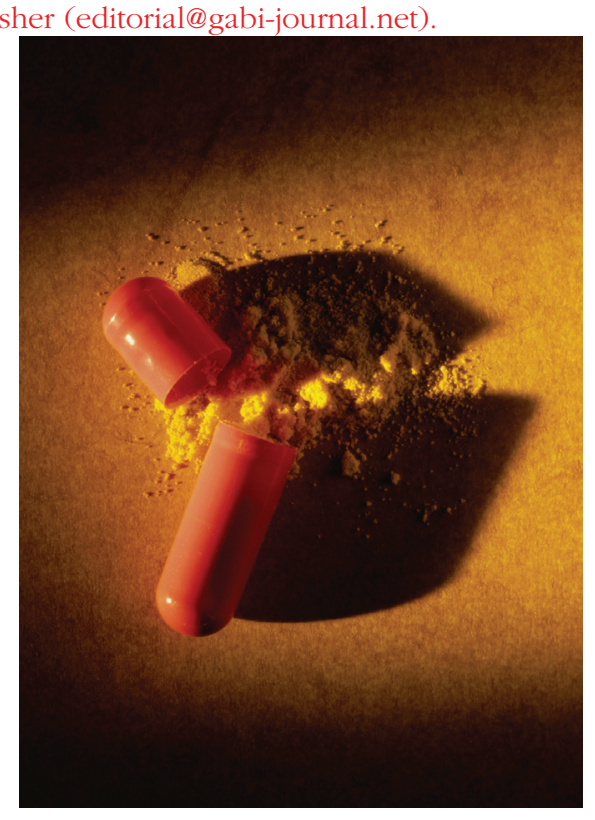

profile, which will mainly be reflected in an altered immunogenicity profile. It is highly expected that adverse events based on the pharmacology of the biological are similar between biosimilar and reference product. Since the manufacturing process of the reference product is proprietary knowledge, the manufacturer of the biosimilar will not be able to precisely replicate the protein product, which may influence the benefit-risk profile [5]. Due to the known limitations of randomized clinical trials [1], and the abbreviated dossier submitted as part of the marketing application for biosimilars, pharmacovigilance is important to obtain (additional) data on the safety profile of biosimilars. From a regulatory perspective, biosimilars have the same pharmacovigilance requirements as their reference products [6]. Biosimilars should, therefore, submit a risk management plan (RMP) as part of their marketing application and should submit periodic safety update reports on a regular basis post-approval.

\section{Routine pharmacovigilance/ spontaneous monitoring}

Routine pharmacovigilance includes the collection of spontaneously reported adverse events by healthcare professionals and patients. Limitations of spontaneous reports of adverse events have been widely acknowledged and described and include under-reporting and a difficult to 
establish causality assessment between the adverse events and the drug of interest $[7,8]$. In the case of biologicals and biosimilars some additional challenges might occur in the assessment of spontaneous reports. The issue of identifiability and naming has been described extensively [9]. Since small changes in the production process can alter the safety profile of a biological it is important that an adverse event can be related to a specific biological product. The new pharmacovigilance legislation stresses the importance of traceability and Member States are obliged to implement activities to improve traceability, including collection of the name of the medicinal product and the batch number [6].

\section{Pro-active risk management}

Since November 2005 applicants are obliged to submit a RMP as part of their marketing application for all new chemical and biological entities, including biosimilars. In the RMP, the safety profile of the medicine has to be described and pharmacovigilance activities should be proposed to further study safety concerns during use of the drug in the real-world setting and, if considered necessary, additional risk minimization activities should be described.

The safety information included in the RMP of the biosimilar should not only be based on the (limited) experience with the biosimilar from the pre-registration trials but should also be based on experience with the reference product. In this way, the RMP of the biosimilar will contain information on the safety profile, which is as complete as possible. In addition, the need for additional efficacy and safety studies in indications in which the biosimilar has not been studied pre-approval, but that are based on extrapolation, should be evaluated on a case-by-case basis. This is also included in the guideline on similar biological medicinal products containing monoclonal antibodies: 1) safety in indications licensed for the reference biological that are claimed based on extrapolation of efficacy and safety data; 2) occurrence of rare and particularly serious adverse events described for the reference product; and 3) detection of novel safety signals [10].

Immunogenicity, including lack of efficacy, is a safety concern that should specifically be addressed in the RMP and the need for additional pharmacovigilance activities should be clearly evaluated. Immunogenicity studies conducted post-approval should be done on a product specific basis and are especially important in case no long-term immunogenicity data has been obtained pre-approval.

Since biologicals are often used in a hospital setting, it can be expected that databases in which mainly GP and public pharmacy data are collected contain only limited information on biologicals. Drug and disease-based registries have shown to be important tools for the post-marketing collection of safety data for biologicals in general $[11,12]$. Biosimilar companies are therefore recommended to participate in already existing registries; this will, for example, improve our knowledge on very rare adverse events like progressive multifocal leukoencephalopathy.

In case there are risk minimization activities in place for the reference product which can be considered a class effect, these risk minimization activities should be included in the RMP of the biosimilar as well.

\section{Conclusions and recommendations}

Pharmacovigilance is an important tool to gain additional knowledge and collect safety data for biologicals and biosimilars due to the limitations of randomized controlled clinical trials. Keywords in the pharmacovigilance of biosimilars are: traceability and pro-active risk management to obtain additional knowledge about the safety of a biosimilar. In this context, immunogenicity is of specific interest and collaboration between companies is encouraged.

\section{For patients}

With the recent patent expiration of some biologicals, companies are able to develop so-called biosimilars of follow-on biologics. Biologicals have a very complex production and purification process which is owned by the company of the reference product. Therefore, the biosimilar might be different from the reference product. Although these small differences are mostly not clinically relevant it might, in rare cases, lead to safety problems. Collection of safety data during use by 'real-patients' is therefore important as well as the traceability of the biological that has been administered if an adverse event develops [13].

\section{Disclaimer}

The views discussed here are personal and not those of the European Medicines Agency, its scientific committees or any other regulatory agency.

\section{Competing interest: None.}

Provenance and peer review: Commissioned; internally peer reviewed.

\section{Co-author}

Sabine MJM Straus, MD, PhD, Medicines Evaluation Board, Utrecht, The Netherlands; Erasmus University Medical Centre, Department of Medical Informatics, Rotterdam, The Netherlands.

\section{References}

1. Stricker BH, Psaty BM. Detection, verification, and quantification of adverse drug reactions. BMJ. 2004;329(7456):44-7.

2. Heemstra EH, Giezen TJ, Mantel-Teeuwisse AK, De Vrueh RLA, Leufkens HGM. Safety-related regulatory actions for orphan drugs in the US and the EU: a cohort study. Drug Saf. 2010;33(2):127-37.

3. Giezen TJ. Risk management of biologicals: a regulatory and clinical perspective [dissertation]. Utrecht, Utrecht Institute for Pharmaceutical Sciences, Division of Pharmacoepidemiology and Clinical Pharmacology, Utrecht University; 2011.

4. Dixon WG, Symmons DP, Lunt M, Watson KD, Hyrich KL, Silman AJ. Serious infections following anti-tumor necrosis factor alpha therapy in patients with rheumatoid arthritis: lessons from interpreting data from observational studies. Arthritis Rheum. 2007;56(9):2896-904.

5. Mellstedt H, Niederwieser D, Ludwig H. The challenge of biosimilars. Ann Oncol. 2008;19(3):411-9.

6. L348/74 Official Journal of the European Union 31.12.2010.

7. Meyboom RH, Egberts AC, Gribnau FW, Hekster YA. Pharmacovigilance in perspective. Drug Saf. 1999;21(6):429-47.

8. Meyboom RH, Hekster YA, Egberts AC, Gribnau FW, Edwards IR. Causal or casual? The role of causality assessment in pharmacovigilance. Drug Saf. 1997;17(6):374-89.

9. Declerck PJ. Biotherapeutics in the era of biosimilars: what really matters is patient safety. Drug Saf. 2007;30(12):1087-92.

10. Committee for Medicinal Products for Human Use. Guideline on similar biological medicinal products containing monoclonal antibodies. London: European Medicines Agency; May 2012.

11. Zink A, Askling J, Dixon WG, Klareskog L, Silman AJ, Symmons DP. European Biologicals registers: methodology, selected results and perspectives. Ann Rheum Dis. 2009;68(8):1240-6.

12. Curtis JR, Jain A, Askling J, et al. A comparison of patient characteristics and outcomes in selected European and U.S. rheumatoid arthritis registries. Semin Arthritis Rheum. 2010;40(1):2-14.e1.

13. Declerck P. Biologicals and biosimilars: a review of the science and its implications. Generics and Biosimilars Initiative Journal (GaBI Journal). 2012;1(2):84-8. doi:10.5639/gabij.2012.0102.018 